[4] A. Belouchrani, K. Abed-Meraim, M. G. Amin, and A. M. Zoubir, "Joint anti-diagonalization for blind source separation," in Proc. IEEE Int. Conf. Acoustics, Speech, Signal Processing (ICASSP), 2001, vol. 5, pp. 2789-2792.

[5] O. Yilmaz and S. Rickard, "Blind separation of speech mixtures via time-frequency masking," IEEE Trans. Signal Process., vol. 52, no. 7, pp. 1830-1847, Jul. 2004.

[6] N. Linh-Trung, A. Belouchrani, K. Abed-Meraim, and B. Boashash, "Separating more sources than sensors using time-frequency distributions," EURASIP J. Appl. Signal Process., vol. 17, pp. 2828-2847, 2005.

[7] A. Aissa-El-Bey, N. Linh-Trung, K. Abed-Meraim, A. Belouchrani, and Y. Grenier, "Underdetermind blind separation of nondisjoint sources in the time-frequency domain," IEEE Trans. Signal Process., vol. 55, no. 3, pp. 897-907, Mar. 2007.

[8] M. V. Hulle, "Clustering approach to square and non-square blind source separation," in Neural Networks for Signal Processing IX_Proc. 1999 IEEE Signal Processing Society Workshop, Aug. 1999, pp. 315-323.

[9] P. Bofill and M. Zibulevsky, "Blind separation of more sources than mixtures using sparsity of their short-time fourier transform," in Proc. ICA, Jun. 2000, pp. 87-92.

[10] P. Bofill and M. Zibulevsky, "Underdetermined blind source separation using sparse representations," Signal Process., vol. 81, no. 11, pp. 2353-2362, Nov. 2001.

[11] Y. Li, A. Cichocki, and S. Amari, "Sparse component analysis for blind source separation with less sensors than sources," in Proc. ICA, Apr. 2003, pp. 89-94.

[12] F. Abrard and Y. Deville, "A time-frequency blind signal separation method applicable to underdetermined mixtures of dependent sources," Signal Process., vol. 85, no. 7, pp. 1389-1403, Jul. 2005.

[13] Y. Deville, M. Benali, and F. Abrard, "Differential source separation for underdetermind instantaneous or convolutive mixtures: Concept and algorithms," Signal Process., vol. 84, pp. 1759-1776, Oct. 2004.

[14] J. Thomas, Y. Deville, and S. Hosseini, "Differential fast fixed-point algorithms for underdetermined instantaneous and convolutive partial blind source separation," IEEE Trans. Signal Process., vol. 55, no. 7, pp. 3717-3729, Jul. 2007.

[15] Y. Deville and M. Puigt, "Temporal and time-frequency correlationbased blind source separation methods. Part I: Determined and underdetermined linear instantaneous mixtures," Signal Process., vol. 87, no. 3, pp. 374-407, Mar. 2007.

[16] G. F. Boudreaux-Bartels and T. W. Parks, "Time-varying filtering and signal estimation using Wigner distribution synthesis techniques," IEEE Trans. Acoust., Speech, Signal Process., vol. ASSP-34, no. 3, pp. 442-451, Jun. 1986.

\section{Precoder Design for Space-Time Coded Systems Over Correlated Rayleigh Fading Channels Using Convex Optimization}

Khoa T. Phan, Sergiy A. Vorobyov, and Chintha Tellambura

\begin{abstract}
A class of computationally efficient linear precoders for spacetime block coded multiple-input multiple-output wireless systems is derived based on the minimization of the exact symbol error rate (SER) and its upper bound. Both correlations at the transmitter and receiver are assumed to be present, and only statistical channel state information in the form of the transmit and receive correlation matrices is assumed to be available at the transmitter. The convexity of the design based on SER minimization is established and exploited. The advantage of the developed technique is its low complexity. We also find various relationships of the proposed designs to the existing precoding techniques, and derive very simple closed-form precoders for special cases such as two or three receive antennas and constant receive correlation. The numerical simulations illustrate the excellent SER performance of the proposed precoders.
\end{abstract}

Index Terms-Convex optimization, correlated fading channels, multiple-input multiple-output (MIMO) systems, precoding, space-time codes.

\section{INTRODUCTION}

Multiple-input multiple-output (MIMO) communication systems are able to mitigate fading and offer significant capacity gains over singleantenna systems [1], [2]. In order to exploit the benefits of MIMO systems, (orthogonal) space-time block codes ((O)STBCs) have been developed [1]. STBC designs presuppose that the channel state information at the transmitter (CSIT) is unavailable [1]. However, if the CSIT is available, the combination of STBCs and precoding techniques can be used to adapt the transmission to the current channel conditions without changing the fixed structures of the transmitter and the receiver [3], [4].

Traditional precoding techniques [3], [4] rely on the full CSIT which can be obtained by exploiting the channel reciprocity for a timedivision duplex (TDD) system and slow variability. However, for rapidly time-varying channel, CSIT can be outdated due to estimation errors and feedback delays. These factors motivate the use of statistical CSIT such as transmit and receive correlation matrices, which vary at a much slower rate than the instantaneous CSIT and may be obtained reliably at the transmitter. Several statistical CSIT based linear precoders have been recently developed (see, for example, [5]-[9] and references therein). However, the emphasis has been on systems with transmit correlation only, assuming that the receive correlation is negligible. The latter assumption may be unrealistic in some cases;

Manuscript received December 07, 2007; revised October 13, 2008. First published October 31, 2008; current version published January 30, 2009. The associate editor coordinating the review of this manuscript and approving it for publication was Prof. Timothy N. Davidson. This work was supported in part by the Natural Sciences and Engineering Research Council (NSERC) of Canada and by the Alberta Ingenuity Foundation, AB, Canada.

K. T. Phan was with the Department of Electrical and Computer Engineering, University of Alberta, Edmonton, AB, Canada. He is now with the Department of Electrical Engineering, California Institute of Technology, Pasadena, CA 91125 USA (e-mail: kphan@caltech.edu).

S. A. Vorobyov and C. Tellambura are with the Department of Electrical and Computer Engineering, University of Alberta, Edmonton, AB T6G 2V4, Canada (e-mail: vorobyov@ece.ualberta.ca; chintha@ece.ualberta.ca).

Color versions of one or more of the figures in this correspondence are available online at http://ieeexplore.iee.org.

Digital Object Identifier 10.1109/TSP.2008.2008563 
for example, when the receiver does not operate in a well-scattered environment [10].

Several performance criteria have been used for precoder design such as the maximum ergodic channel capacity [7] and the minimum (Chernoff bound) pairwise error probability (PEP) [9]. Alternatively, the minimum of the exact symbol error rate (SER) has been used in [8] for the case of transmit correlation only and generalized in [11] for the case of both transmit and receive correlations. However, [11] neither recognizes nor exploits the convexity of the minimum SER precoder design problem.

This correspondence considers the problem of SER minimization for precoding design for OSTBC systems ${ }^{1}$ over correlated Rayleigh-fading channels, just as in [11]. However, this correspondence concerns of the algorithmic and complexity issues of precoding design. The objectives of this work are i) to establish the convexity result for the SER minimization problem; ii) to develop a low complexity guaranteed-convergence iterative algorithm for optimal precoder design; iii) to develop low complexity precoders for a number of special cases of particular interest; and iv) to show the relationships to the existing precoder designs. ${ }^{2}$

\section{SYSTEM MODEL}

Consider a communication system that employs OSTBC and linear precoding at the transmitter. The input of the space-time encoder is a $Q \times 1$ vector $\mathbf{s} \triangleq\left[s_{1}, s_{2}, \ldots, s_{Q}\right]^{T}$ of information-bearing symbols chosen from different signal constellations with $E\left[\left|s_{i}\right|^{2}\right]=1$ where $(\cdot)^{T}$ and $E[\cdot]$ denote the transpose and statistical expectation, respectively. The output of the space-time encoder and precoder is transmitted over the correlated Rayleigh fading MIMO channel. Then the $N_{\mathrm{r}} \times T$ matrix of received signal samples can be written as

$$
\mathbf{Y}=\sqrt{\frac{E_{s}}{N_{\mathrm{t}}}} \mathbf{H F C}+\mathbf{Z}
$$

where $N_{\mathrm{r}}$ is the number of receive antennas, $E_{s}$ is the transmit power, $T$ is the number of time periods used for transmitting one block of symbols, $\mathbf{C}$ is the $N_{\mathrm{t}} \times T$ OSTBC matrix, $\mathbf{F}$ is the $N_{\mathrm{t}} \times N_{\mathrm{t}}$ precoding matrix, $\mathbf{H}$ is the $N_{\mathrm{r}} \times N_{\mathrm{t}}$ channel matrix, and $\mathbf{Z}$ is the $N_{\mathrm{r}} \times T$ matrix of independent identically distributed (i.i.d.) zero-mean circularly symmetric complex additive white Gaussian noise (AWGN) samples with variance $N_{0}$, i.e., $[\mathbf{Z}]_{i j} \sim \mathcal{C N}\left(0, N_{0}\right)$.

The symbol rate of the OSTBC is $K \triangleq Q / T$, i.e., $T$ time periods are used to transmit $Q$ symbols. It has been shown in [1] that if OSTBCs are used, the spatial matched filtering does not color the AWGN and decouples the MIMO channel into $Q$ parallel channels with effective symbol instantaneous signal-to-noise ratio (SNR) $\gamma=\left(E_{s} / K N_{\mathrm{t}} N_{0}\right) \alpha$ where $\alpha \triangleq\|\mathbf{H F}\|_{F}^{2}$ and $\|\cdot\|_{F}^{2}$ denotes the Frobenius norm of matrix.

The channel correlation matrix $\mathbf{R} \triangleq E\left[\operatorname{vec}(\mathbf{H}) \operatorname{vec}^{H}(\mathbf{H})\right]$ is assumed to be known at the transmitter. Here $\operatorname{vec}(\cdot)$ and $(\cdot)^{H}$ stand for the matrix vectorization operation and Hermitian transpose, respectively. In the well known Kronecker correlation model, the channel matrix $\mathbf{H}$ can be written as $\mathbf{H}=\mathbf{R}_{\mathrm{r}}^{1 / 2} \tilde{\mathbf{H}} \mathbf{R}_{\mathbf{t}}^{1 / 2}$, where $\mathbf{R}_{\mathrm{t}}$ and $\mathbf{R}_{\mathrm{r}}$ are the transmit and receive correlation matrices of sizes $N_{\mathrm{t}} \times N_{\mathrm{t}}$ and $N_{\mathrm{r}} \times N_{\mathrm{r}}$, respectively, and $\tilde{\mathbf{H}}$ consists of i.i.d. $\mathcal{C N}(0,1)$ elements [16]. Consequently, the channel correlation matrix $\mathbf{R}$ is the Kronecker product of the transmit and receive correlation matrices $\mathbf{R}=\mathbf{R}_{\mathrm{r}}^{T} \otimes \mathbf{R}_{\mathrm{t}}$.

${ }^{1}$ Note that extension of the minimum SER based precoding designs to the systems with quasi-orthogonal STBCs [12] and linear dispersion codes [13] is also of interest, but such extension is outside of the scope of this correspondence.

${ }^{2} \mathrm{~A}$ preliminary version of this work has been presented in conference contribution [14].

\section{PRECODER DESIGN PROBLEM}

\section{A. Exact SER, Precoder Design Problem, and Its Convexity}

Given the receive instantaneous SNR $\gamma$, the SER in the case of, for example, $M$-ary pulse amplitude modulation ${ }^{3}$ (M-PAM) can be evaluated as [17]

$$
\mathrm{SER}_{\mathrm{PAM}}^{\gamma}=\frac{2}{\pi} \frac{M-1}{M} \int_{0}^{\frac{\pi}{2}} \exp \left(-\frac{g_{\mathrm{PAM}} \gamma}{\sin ^{2} \phi}\right) d \phi
$$

where $g_{\text {PAM }} \triangleq 3 /\left(M^{2}-1\right)$.

Using the moment-generating function (MGF) approach, the exact SER can be written for general and Kronecker correlation cases as [8], [11]

$$
\begin{aligned}
\operatorname{SER}_{\mathrm{PAM}} & =\frac{2(M-1)}{\pi M} \int_{0}^{\frac{\pi}{2}} \operatorname{det}\left(\mathbf{I}+\frac{\tilde{g}}{\sin ^{2} \phi}\left(\mathbf{I} \otimes \mathbf{F F} \mathbf{F}^{H}\right) \mathbf{R}\right)^{-1} d \phi \\
& =\frac{2(M-1)}{\pi M} \int_{0}^{\frac{\pi}{2}} \operatorname{det}\left(\mathbf{I}+\frac{\tilde{g}}{\sin ^{2} \phi} \mathbf{R}_{\mathbf{r}} \otimes\left(\mathbf{F F} \mathbf{F}^{H} \mathbf{R}_{\mathbf{t}}\right)\right)^{-1} d \phi
\end{aligned}
$$

where $\tilde{g} \triangleq g_{\mathrm{PAM}} E_{s} /\left(K N_{\mathrm{t}} N_{0}\right)$ and $\operatorname{det}(\cdot)$ denotes the determinant of matrix.

The precoder design problem can now be posed as the following SER minimization problem [11]:

$$
\min _{\tilde{\mathbf{F}}} \text { SER }_{\text {PAM }} \quad \text { subject to } \operatorname{Tr}(\tilde{\mathbf{F}})=1, \quad \tilde{\mathbf{F}} \succcurlyeq \mathbf{0}
$$

where $\tilde{\mathbf{F}} \triangleq \mathbf{F F}^{H}$ is a new optimization variable, and the constraint $\operatorname{Tr}(\tilde{\mathbf{F}})=1$ guarantees that the average transmit power is constant. ${ }^{4}$ Since $\mathbf{F}$ is a square matrix of any rank, the optimal $\tilde{\mathbf{F}}$ can also be of any rank, and optimization over $\tilde{\mathbf{F}}$ is equivalent to optimization over $\mathbf{F}$. The optimal precoding matrix $\mathbf{F}_{\text {opt }}$ can be found then as $\mathbf{F}_{\text {opt }}=\tilde{\mathbf{F}}_{\text {opt }}^{1 / 2}$.

The following theorem is in order.

Theorem 1: The optimization problem (5) is convex on $\tilde{\mathbf{F}} \succcurlyeq \mathbf{0}$.

Proof: For the general correlation case (3), let $\mathbf{V}(\tilde{\mathbf{F}}) \triangleq$ $\mathbf{I}+\left(\tilde{g} / \sin ^{2} \phi\right)(\mathbf{I} \otimes \tilde{\mathbf{F}}) \mathbf{R}$. We observe that $\operatorname{det}\left(\mathbf{V}^{-1}(\tilde{\mathbf{F}})\right)=$ $\exp (-\log \operatorname{det}(\mathbf{V}(\tilde{\mathbf{F}})))$. It is well known that the function $-\log \operatorname{det}(\mathbf{V})$ is convex on $\mathbf{V} \geqslant 0$ [15, p. 74]. Therefore, in order to prove that $-\log \operatorname{det}(\mathbf{V}(\tilde{\mathbf{F}}))$ is convex on $\tilde{\mathbf{F}} \geqslant \mathbf{0}$ for fixed $\phi$, it is sufficient to prove that the matrix function $\tilde{\mathbf{V}}(\tilde{\mathbf{F}}) \triangleq(\mathbf{I} \otimes \tilde{\mathbf{F}}) \mathbf{R}$ is affine on $\tilde{\mathbf{F}}$ which is obviously true since $\tilde{\mathbf{V}}(\tilde{\mathbf{F}})$ is a linear function of $\tilde{\mathbf{F}}$. Thus, $-\log \operatorname{det}(\mathbf{V}(\tilde{\mathbf{F}}))$ is convex. Since the exponential function is convex and monotonically increasing, the function $\exp (-\log \operatorname{det}(\mathbf{V}(\tilde{\mathbf{F}})))$ is convex on $\tilde{\mathbf{F}}$, and we conclude that the function $\operatorname{det}\left(\mathbf{V}^{-1}(\tilde{\mathbf{F}})\right)$ is also convex on $\tilde{\mathbf{F}}$. Observing that the integral in (3) can be seen as a sum of convex terms, we conclude that the objective function of the problem (5) is a convex function ${ }^{5}$ of $\tilde{\mathbf{F}}$. The constraints in (5) are also convex on $\tilde{\mathbf{F}}$. This completes the proof.

Although the convexity of the worst-case PEP minimization based precoder has been shown in [5], the convexity of the problem (5) has not been recognized before. Since it is convex, the problem (5) can be solved using well-established interior-point methods. However, the

${ }^{3}$ Note that the cases of $\boldsymbol{M}$-ary quadrature amplitude modulation (M-QAM), and $\boldsymbol{M}$-ary phase-shift keying (M-PSK) can be adopted straightforwardly, and therefore, are omitted for the sake of brevity.

${ }^{4}$ Since the optimal precoder depends on the SNR via $\tilde{\boldsymbol{g}}$, the optimal precoding matrix must be recomputed for different SNR conditions.

${ }^{5}$ Note that the result holds straightforwardly for the Kronecker correlation case (4) as well. 
gradient computation involves inversion of $N_{\mathrm{t}} N_{\mathrm{r}} \times N_{\mathrm{t}} N_{\mathrm{r}}$ matrix and evaluation of $N_{\mathrm{t}}^{2}$ integrals that significantly increases the overall computational complexity of the problem.

\section{B. Reduced-Complexity Design}

For the Kronecker correlation case, let us consider the eigenvalue decomposition (EVD) of the correlation matrices $\mathbf{R}_{\mathrm{t}}$ and $\mathbf{R}_{\mathrm{r}}$ given by

$$
\begin{aligned}
& \mathbf{R}_{\mathrm{t}}=\mathbf{U}_{\mathrm{t}} \boldsymbol{\Sigma}_{\mathrm{t}} \mathbf{U}_{\mathrm{t}}^{H} \\
& \mathbf{R}_{\mathrm{r}}=\mathbf{U}_{\mathrm{r}} \boldsymbol{\Sigma}_{\mathrm{r}} \mathbf{U}_{\mathrm{r}}^{H}
\end{aligned}
$$

where $\mathbf{U}_{\mathrm{t}}, \mathbf{U}_{\mathrm{r}}$ are the matrices of eigenvectors, and $\boldsymbol{\Sigma}_{\mathrm{t}} \triangleq$ $\operatorname{diag}\left(\lambda_{1}, \ldots, \lambda_{N_{\mathrm{t}}}\right), \boldsymbol{\Sigma}_{\mathrm{r}} \triangleq \operatorname{diag}\left(\chi_{1}, \ldots, \chi_{N_{\mathrm{r}}}\right)$ are the diagonal eigenvalue matrices of the transmit and receive correlation matrices, respectively.

Substituting (6) and (7) into (5), the precoder design problem can be written as

$$
\begin{aligned}
& \min _{\overline{\mathbf{F}}} \int_{0}^{\frac{\pi}{2}} \operatorname{det}\left(\mathbf{I}+\frac{\tilde{g}}{\sin ^{2} \phi} \boldsymbol{\Sigma}_{\mathrm{r}} \otimes\left(\boldsymbol{\Sigma}_{\mathrm{t}}^{1 / 2} \overline{\mathbf{F}} \boldsymbol{\Sigma}_{\mathrm{t}}^{1 / 2}\right)\right)^{-1} d \phi \\
& \text { subject to } \operatorname{Tr}(\overline{\mathbf{F}})=1, \quad \overline{\mathbf{F}} \succcurlyeq \mathbf{0}
\end{aligned}
$$

where $\overline{\mathbf{F}} \triangleq \mathbf{U}_{\mathbf{t}}^{H} \mathbf{F} \mathbf{F}^{H} \mathbf{U}_{\mathrm{t}}$ is the new optimization variable. The average power constraint is now expressed in terms of $\overline{\mathbf{F}}$, and it holds true because the matrix $\mathbf{U}_{\mathrm{t}}$ is unitary. The optimal precoding matrix $\mathbf{F}_{\text {opt }}$ can be found as $\mathbf{F}_{\text {opt }}=\mathbf{U}_{\mathrm{t}} \overline{\mathbf{F}}_{\text {opt }}^{1 / 2}$.

Observation 1: It can be seen from (8) that the transmit and receive correlations have different effects on the design of the linear precoder. Particularly, the precoder matrix depends on the receive correlation matrix $\mathbf{R}_{\mathrm{r}}$ only through its eigenvalues $\boldsymbol{\Sigma}_{\mathrm{r}}$, while it depends on both eigenvalues $\boldsymbol{\Sigma}_{\mathrm{t}}$ and eigenvectors $\mathbf{U}_{\mathrm{t}}$ of $\mathbf{R}_{\mathrm{t}}$. Therefore, the optimal precoder mainly exploits the transmit correlation information.

Exploiting the fact that the optimal matrix $\overline{\mathbf{F}}$ in (8) is diagonal [9], [11] and replacing the diagonal elements of $\overline{\mathbf{F}}$ by $\mathbf{x} \triangleq\left[x_{1}, \ldots, x_{N_{\mathrm{t}}}\right]=$ $\operatorname{diag}(\overline{\mathbf{F}}) \in \mathcal{R}_{+}^{N_{\mathrm{t}}}$, the problem (8) can be simplified to the following optimization problem

$$
\begin{aligned}
& \min _{\mathbf{x}} \int_{0}^{\frac{\pi}{2}} \prod_{i=1}^{N_{\mathrm{r}}} \prod_{j=1}^{N_{\mathrm{t}}}\left(1+\frac{\tilde{g}}{\sin ^{2} \phi} \chi_{i} \lambda_{j} x_{j}\right)^{-1} d \phi \\
& \text { subject to } \sum_{j=1}^{N_{\mathrm{t}}} x_{j}=1, x_{j} \geq 0, j=1, \ldots, N_{\mathrm{t}} .
\end{aligned}
$$

Thus, the optimization problem (5) with matrix variable $\tilde{\mathbf{F}} \succcurlyeq 0$ is simplified into the optimization problem (9) with vector variable $\mathbf{x} \in$ $\mathcal{R}_{+}^{N_{t}}$. The problem (9) is convex on $\mathbf{x} \in \mathcal{R}_{+}^{N_{t}}$ due to the fact that its objective function is the product of convex decreasing and positive functions $\left(1+\left(\tilde{g} / \sin ^{2} \phi\right) \chi_{i} \lambda_{j} x_{j}\right)^{-1}$, and thus, is also convex [15]. The computational complexity of solving the problem (9) is lower than that of the problem (5).

Although the aforementioned dimension reduction technique for precoder design in the Kronecker correlation case has been widely used before (see, for example, [11]), the newly established convexity result allows to develop the efficient guaranteed-convergent logarithmic barrier function based gradient descent algorithm which is summarized Appendix A. ${ }^{6}$ The polynomial complexity of such algorithm is mostly due to the gradient computation for the objective function in (9). The convergence and complexity analysis of the algorithm and the comparison to the precoder of [11] it terms of complexity are presented in Appendix B.

${ }^{6}$ Note that a "better" step direction than the gradient descent direction can help to reduce the number of iterations when solving the problem (9). However, for brevity, we do not explore this avenue.

\section{Single-Receive-Antenna Case}

In practice, the receiver is often equipped with a single antenna, especially in the downlink mode when the mobile handset is size-limited. The SER minimization based precoder in the case of a single receive antenna is developed in [8], but the solution is provided only for the SER upper bound. The exact problem formulation in this case follows from (9) (a single product is left in the objective function). Moreover, using the equation $(1 / \pi) \int_{0}^{\pi / 2} \prod_{j=1}^{L}\left(\sin ^{2} \theta / \sin ^{2} \theta+c_{j}\right) d \theta=$ $(1 / 2) \sum_{j=1}^{L}\left(1-\sqrt{c_{j} / 1+c_{j}}\right) \prod_{\substack{n=1 \\ n \neq j \\ n \neq j}}^{L}\left(c_{j} / c_{j}-c_{n}\right)$ (see [17]), the objective function can be expressed in closed-form that further reduces the complexity of the gradient descent method because the gradient can be computed then easily without any integration involved. This design for single-antenna receiver can be seen as a completion of the work in [8], especially in terms of the design complexity.

\section{LOW-COMPLEXITY LINEAR PRECODERS}

\section{A. Upper Bound Problem}

Replacing $\sin (\phi)$ in (9) by its maximum value ${ }^{7} \sin (\pi / 2)=1$, the objective function in (9) can be upper bounded as $\prod_{i=1}^{N_{\mathrm{r}}} \prod_{j=1}^{N_{\mathrm{t}}}(1+$ $\left.\tilde{g} \chi_{i} \lambda_{j} x_{j}\right)^{-1}$. The variables of this function are nonseparable. Observing that the logarithmic function $\log (x)$ is monotonically increasing for nonnegative $x$, the upper bound of the objective function in (9) can be rewritten as a sum instead of the product, and the following upper bound optimization problem can be obtained:

$$
\begin{aligned}
& \min _{\mathbf{x}} \sum_{j=1}^{N_{\mathrm{t}}} \sum_{i=1}^{N_{\mathrm{r}}}-\log \left(1+\tilde{g} \chi_{i} \lambda_{j} x_{j}\right) \\
& \text { subject to } \quad \sum_{j=1}^{N_{\mathrm{t}}} x_{j} \leq 1, \quad x_{j} \geq 0, j=1, \ldots, N_{\mathrm{t}} .
\end{aligned}
$$

The fact that the objective function is a decreasing function of $\mathrm{x} \in$ $\mathcal{R}_{+}^{N_{\mathrm{t}}}$ has been also used to relax the equality constraint $\sum_{j=1}^{N_{\mathrm{t}}} x_{j}=$ 1 to inequality. The equality will be always achieved for the optimal solution; that is, all available power will be loaded along eigenbeams to achieve the system's best performance.

Faster (than interior-point methods) computational procedures can be developed for (10) using the monotonic structure of the problem additionally to its convexity. Indeed, due to separability of the variables in the objective function, the theory of Lagrange dual decomposition can be applied. The Lagrangian for the optimization problem (10) is given by

$$
\begin{aligned}
\mathcal{L}(\mathbf{x}, \mu)= & \sum_{j=1}^{N_{\mathrm{t}}} \sum_{i=1}^{N_{\mathrm{r}}}-\log \left(1+\tilde{g} \chi_{i} \lambda_{j} x_{j}\right) \\
& +\mu\left(\sum_{j=1}^{N_{\mathrm{t}}} x_{j}-1\right)-\sum_{j=1}^{N_{\mathrm{t}}} \mu_{j} x_{j}=\sum_{j=1}^{N_{\mathrm{t}}} \tilde{f}\left(x_{j}\right)-\mu
\end{aligned}
$$

where $\mu \geq 0, \mu_{j} \geq 0, j=1, \ldots, N_{\mathrm{t}}$ are the Lagrange multipliers and $\tilde{f}\left(x_{j}\right) \triangleq \sum_{i=1}^{N_{\mathrm{r}}}-\log \left(1+\tilde{g} \chi_{i} \lambda_{j} x_{j}\right)+\mu x_{j}-\mu_{j} x_{j}, j=1, \ldots, N_{\mathrm{t}}$. The optimal solution of the optimization problem (10) and the Lagrange multipliers must satisfy the following Karush-Kuhn-Tucker (KKT) conditions

$$
\begin{aligned}
& \frac{\partial \mathcal{L}(\mathbf{x}, \mu)}{\partial \mathbf{x}}=\sum_{i=1}^{N_{\mathrm{r}}} \frac{1}{x_{j}+\left(\tilde{g} \chi_{i} \lambda_{j}\right)^{-1}}-\mu+\mu_{j}=0 \\
& \frac{\partial \mathcal{L}(\mathbf{x}, \mu)}{\partial \mu_{j}}=\mu_{j} x_{j}=0, \quad j=1, \ldots, N_{\mathrm{t}}
\end{aligned}
$$

${ }^{7}$ Setting $\sin (\phi)=1$ in this way generates the standard Chernoff bound on the complementary error function. 


$$
\frac{\partial \mathcal{L}(\mathbf{x}, \mu)}{\partial \mu}=\mu \sum_{j=1}^{N_{\mathrm{t}}} x_{j}=1 .
$$

Then, the optimal solution of (10) can be found in the form

$$
x_{j}^{*}=\left[x_{j}(\mu)\right]^{+}:=\max \left\{x_{j}(\mu), 0\right\}, \quad j=1, \ldots, N_{\mathrm{t}}
$$

where $x_{j}(\mu), j=1, \ldots, N_{\mathrm{t}}$ are the solutions of the KKT equations and $\mu$ is the solution of the equation

$$
\sum_{j=1}^{N_{\mathrm{t}}} x_{j}^{*}=\sum_{j=1}^{N_{\mathrm{t}}}\left[x_{j}(\mu)\right]^{+}=1 .
$$

It follows from the KKT conditions that it is not guaranteed for the optimal solution that $x_{j}>0, \forall j$. The non-increasing order of the eigenvalues of $\mathbf{R}_{\mathrm{t}}$ implies that $x_{1} \geq x_{2} \ldots \geq x_{N_{\mathrm{t}}}$. Then the number $\bar{N}_{\mathrm{t}}$ of positive $x_{j}$ can be found by first setting $\bar{N}_{\mathrm{t}}=N_{\mathrm{t}}$ and checking if $x_{\bar{N}_{\mathrm{t}}}>0$. If this is the case, $x_{j}, \forall j$ are found by just computing (15) and (16). Otherwise, we set $x_{\bar{N}_{\mathrm{t}}}=0$ and go to the next iteration by replacing $\bar{N}_{\mathrm{t}}$ with $\bar{N}_{\mathrm{t}}-1$. Interestingly, this process is similar to that of the water-filling rule. The algorithm for finding the optimal solution of (10) is summarized in Appendix C.

\section{B. Two Receive Antennas}

In this case, the receive correlation matrix has two eigenvalues $\chi_{1}$ and $\chi_{2}$. The globally optimal solution to the problem (10) can be found by solving the system of KKT equations (12)-(14). Introducing a new variable $\bar{\mu} \triangleq 1 / \mu$, the following second order equation is obtained

$$
x_{j}^{2}+\left(\tilde{g}_{1 j}+\tilde{g}_{2 j}-2 \bar{\mu}\right) x_{j}+\tilde{g}_{1 j} \tilde{g}_{2 j}-\bar{\mu}\left(\tilde{g}_{1 j}+\tilde{g}_{2 j}\right)=0, j=1, \ldots N_{\mathrm{t}}
$$

where $\tilde{g}_{1 j} \triangleq 1 /\left(\tilde{g} \chi_{1} \lambda_{j}\right)$ and $\tilde{g}_{2 j} \triangleq 1 /\left(\tilde{g} \chi_{2} \lambda_{j}\right)$. The solution of (17) can be found as

$$
\begin{aligned}
x_{j}^{*}=\frac{1}{2}\left[-\tilde{g}_{1 j}-\right. & \tilde{g}_{2 j}+2 \bar{\mu} \\
& \left.+\sqrt{\left(\tilde{g}_{1 j}-1 \tilde{g}_{2 j}\right)^{2}+4 \bar{\mu}^{2}}\right]^{+}, j=1, \ldots, N_{\mathrm{t}} .
\end{aligned}
$$

Then, $\bar{\mu}$ is chosen such that it satisfies the condition (16). Although, the (16) cannot be solved for $\bar{\mu}$ in closed form, a procedure similar to the iterative bisection procedure (IBP) in the end of Appendix C which makes use of the fact that the left-hand side of (16) is increasing function of $\bar{\mu}$ can be adopted.

\section{Three Receive Antennas}

In this case, the receive correlation matrix has three eigenvalues $\chi_{1}$, $\chi_{2}$, and $\chi_{3}$. Similar to the two receive antennas case, the following third-order equation is obtained:

$$
x_{j}^{3}+a_{2} x_{j}^{2}+a_{1} x_{j}+a_{0}=0, \quad j=1, \ldots N_{\mathrm{t}}
$$

where $a_{2} \triangleq \tilde{g}_{1 j}+\tilde{g}_{2 j}+\tilde{g}_{3 j}-3 \bar{\mu}, a_{1} \triangleq \tilde{g}_{1 j} \tilde{g}_{2 j}+\tilde{g}_{1 j} \tilde{g}_{3 j}+\tilde{g}_{2 j} \tilde{g}_{3 j}-$ $2 \bar{\mu}\left(\tilde{g}_{1 j}+\tilde{g}_{2 j}+\tilde{g}_{3 j}\right), a_{0} \triangleq \tilde{g}_{1 j} \tilde{g}_{2 j} \tilde{g}_{3 j}-\bar{\mu}\left(\tilde{g}_{1 j} \tilde{g}_{2 j}+\tilde{g}_{1 j} \tilde{g}_{3 j}+\tilde{g}_{2 j} \tilde{g}_{3 j}\right)$, $\tilde{g}_{1 j} \triangleq 1 /\left(\tilde{g} \chi_{1} \lambda_{j}\right), \tilde{g}_{2 j} \triangleq 1 /\left(\tilde{g} \chi_{2} \lambda_{j}\right)$, and $\tilde{g}_{3 j} \triangleq 1 /\left(\tilde{g} \chi_{3} \lambda_{j}\right)$. Let $q \triangleq(1 / 3) a_{1}-(1 / 9) a_{2}^{2}, r \triangleq(1 / 6)\left(a_{1} a_{2}-3 a_{0}\right)-(1 / 27) a_{2}^{3}, s_{1} \triangleq$ $\left[r+\left(q^{3}+r^{2}\right)^{1 / 2}\right]^{1 / 2}$, and $s_{2} \triangleq\left[r-\left(q^{3}+r^{2}\right)^{1 / 2}\right]^{1 / 2}$. Due to the convexity of our problem, there is only one real solution, that is

$$
x_{j}^{*}=\left[\left(s_{1}+s_{2}\right)-a_{2} / 3\right]^{+}, \quad j=1, \ldots, N_{\mathrm{t}} .
$$

Then, $\bar{\mu}$ is chosen such that $\sum_{j=1}^{N_{\mathrm{t}}} x_{j}^{*}=\left[\left(s_{1}+s_{2}\right)-a_{2} / 3\right]^{+}=$ 1. As before, a one-dimensional search can be carried out to find the optimal $\bar{\mu}$.

\section{Constant Receive Correlation}

A popular constant correlation model [10] can be viewed as an approximation for the case of closely spaced antennas. ${ }^{8}$ Assuming a constant correlation model at the receiver with correlation coefficient $\rho \in$ $[0,1)$, the receive correlation matrix $\mathbf{R}_{\mathrm{r}}$ has one eigenvalue of order one equal to $\chi_{1}=\rho\left(N_{\mathrm{r}}-1\right)+1$, and one eigenvalue of order $N_{\mathrm{r}}-1$ equal to $\chi_{2}=1-\rho$. Thus, the case of constant receive correlation for arbitrary number of receive antennas is mathematically equivalent to the case of two receive antennas.

\section{E. Transmit-Side Correlation Only}

In this case, the receive correlation matrix is an identity matrix, ${ }^{9}$ and the objective function of (10) simplifies to $\sum_{j=1}^{N_{\mathrm{t}}}-\log \left(1+\tilde{g} \lambda_{j} x_{j}\right)$. By solving the system of KKT conditions, it is easy to find that the optimal $\mathbf{x}$ is given by the following water-filling procedure:

$$
x_{j}^{*}=\left[\mu^{-1}-\left(\tilde{g} \lambda_{j}\right)^{-1}\right]^{+}, \quad j=1, \ldots, N_{\mathrm{t}}
$$

and $\mu$ is chosen such that $\sum_{j=1}^{N_{\mathrm{t}}} x_{j}^{*}(\mu)=1$. This result agrees with the existing results for the case of transmit correlation only [6], [8].

\section{Simulation Results}

A precoded OSTBC MIMO systems with various number of transmit and receive antennas is considered in the simulation examples which follow. The 4-PAM constellation is used and the transmit power $E_{s}=$ $N_{\mathrm{t}}$. The performance is shown in terms of the SER, and a total of $10^{5}$ runs is used to obtain each point of the SER curves.

The first example studies a joint transmit-receive correlated system with two transmit and two receive antennas, employing the Alamouti code. The correlation coefficients between different antennas are $\rho_{\mathrm{t}}=$ $\rho_{\mathrm{r}}=0.6$. Four different precoders are considered: trivial precoder $(\mathbf{F}=\mathbf{I}$, or no precoding), the minimum SER precoder (9), the minimum SER upper-bound precoder (10), and the worst-case PEP-based precoder of [6] and [9]. Note that the precoder of [11] performs similarly to the precoder (9) if fixed-point iterations converge to the globally optimal solution.

Fig. 1 displays the SER versus SNR of the aforementioned precoders. The proposed precoders outperform the trivial precoder, especially in the low to medium SNRs regions. Particularly, the performance gain of the proposed precoders over the trivial precoder is about $1.8 \mathrm{~dB}$ for the SER of $10^{-2}$. Almost identical performance is observed for both the minimum SER upper-bound and the minimum exact SER precoders. However, the former has a lower complexity than the latter, and thus, is recommended for practical implementation. The minimum SER upper-bound precoder performs slightly better than that the worst-case PEP-based precoder of [9], especially in the medium to high SNR regions. The performance of all precorders is asymptotically identical to the trivial precoder in the high SNR region. This observation agrees with the intuition that in this region, transmitter optimization provides little benefit, but can not worsen the performance. It is because the term $\tilde{g}$ in (9) and (10) dominates the eigenvalues of the correlation matrices when SNR is large and the number of antennas is small. In this case, the system performance does not depend significantly on the precoding matrix.

In our second example, a single-receive-antenna system employing the real OSTBC for four transmit antennas (see [1] for the code matrix) is considered. The same precoders as in the previous example are compared. The exponential correlation model [10] is used with correlation coefficient between the neighboring antennas $\rho_{\mathrm{t}}=0.6$.

${ }^{8}$ The case of closely spaced antennas is typical for downlink mode. It represents the worst (least favorable) antenna configuration.

${ }^{9}$ It is applicable when either the receiver has one antenna, or there is a rich scattering environment at the receiver side. 


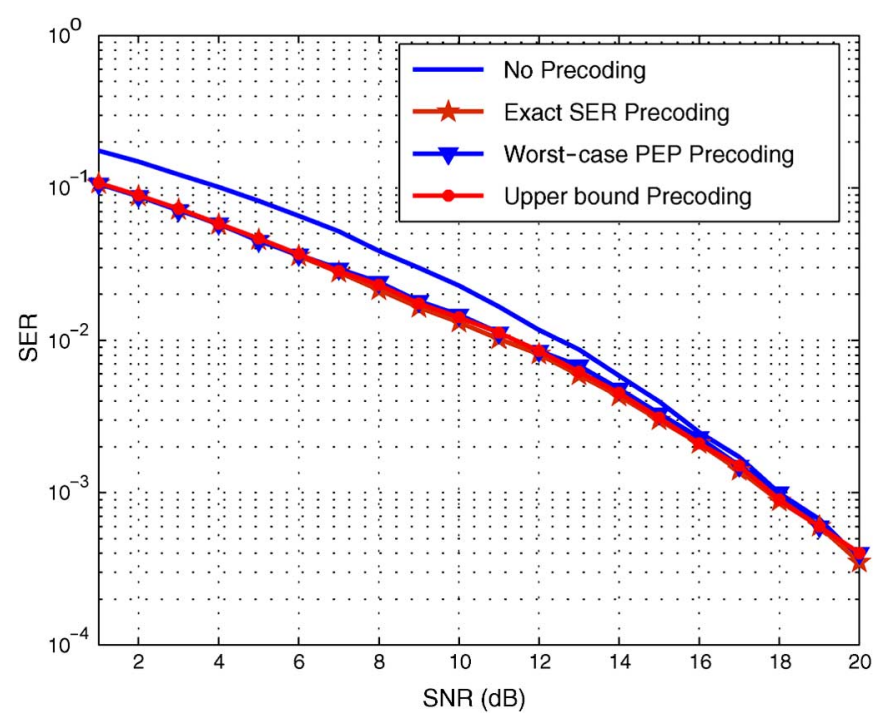

Fig. 1. SER performance of the Alamouti coded MIMO system with transmit and receive correlations $\rho_{\mathrm{r}}=\rho_{\mathrm{t}}=\mathbf{0 . 6}$.

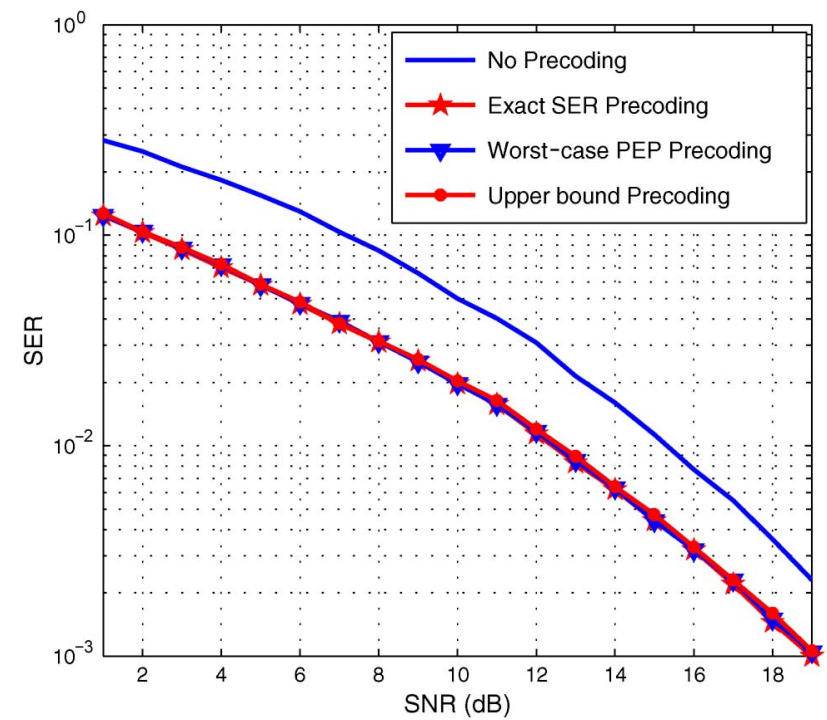

Fig. 2. SER performance of the system with four transmit antennas and singlereceive antenna and transmit exponential correlation $\boldsymbol{\rho}_{\mathrm{t}}=\mathbf{0 . 6}$.

Fig. 2 shows the SER versus SNR. It can be seen that the proposed precoders significantly outperform the trivial precoder. For example, the performance gain of the proposed precoders is about $3.5 \mathrm{~dB}$ at the SER of $8 \cdot 10^{-3}$. Moreover, the performance gain does not decrease as the SNR increases, which agrees also with the results reported in [6].

Finally, we investigate the SER of the proposed precoders versus the transmit and receive correlations for the two transmit two receive antenna system. Specifically, the performance of the precoder (10) is compared to that of the trivial precoder for an SNR of $12 \mathrm{~dB}$. Fig. 3 shows the SER versus correlation coefficient at the receiver for fixed transmit correlations $\rho_{\mathrm{t}}=0.6$ and $\rho_{\mathrm{t}}=0.2$. The proposed precoders improve the system performance when the receive correlation coefficient is small. As the coefficient increases, the performance of both schemes decreases. Moreover, both the trivial precoder and the proposed precoder show similar performance for all values of the receive correlation if the transmit correlation is small $\left(\rho_{\mathrm{t}}=0.2\right)$. This observation agrees with a well-known result that in the case of receive correlation only, the optimal precoding strategy is a simple equal power loading (trivial precoding). Fig. 4 displays the SER versus the correlation coefficient at the transmitter for fixed correlations at the receiver $\rho_{\mathrm{r}}=0.6$ and $\rho_{\mathrm{r}}=0.2$. The performance gap between the proposed

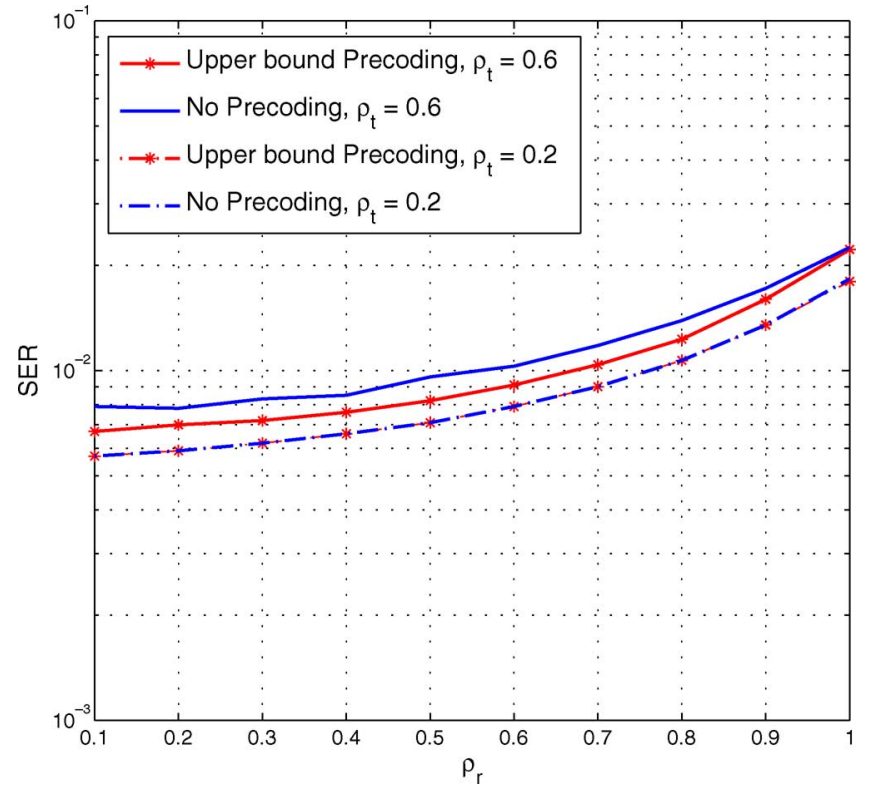

Fig. 3. SER performance of the Alamouti coded MIMO system with constant transmit correlation, receive correlation $\boldsymbol{\rho}_{\mathrm{r}}=[0.1,1], \mathbf{S N R}=12 \mathrm{~dB}$.

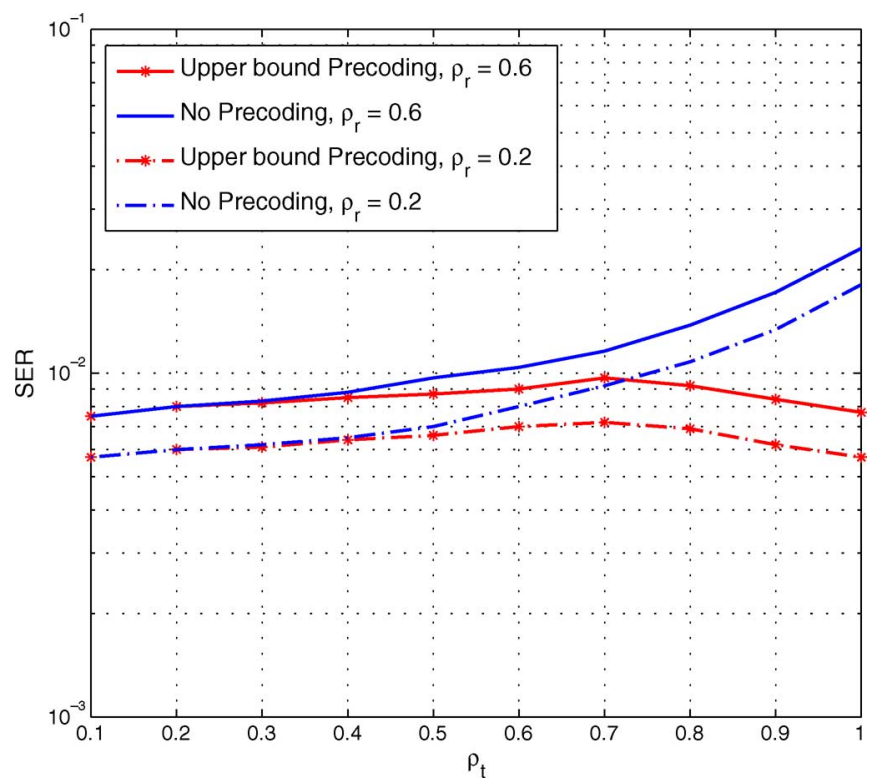

Fig. 4. SER performance of the Alamouti coded MIMO system with constant receive correlation, transmit correlation $\boldsymbol{\rho}_{\mathrm{t}}=[\mathbf{0 . 1}, \mathbf{1}], \mathbf{S N R}=12 \mathrm{~dB}$.

precoder and the trivial precoder is negligible when the transmit correlation is low, and increases when the correlation increases. Therefore, the proposed precoder mostly exploits the transmit correlation in order to reduce the SER which confirms the theoretical Observation 1.

\section{CONCLUSION}

A convex optimization based precoder design for OSTBC MIMO systems over Rayleigh fading channels with both transmit and receive correlations has been presented, and optimal statistical CSIT based precoders have been derived. The design aims at minimizing the exact SER or the Chernoff bound on the exact SER. The convex formulation facilitates the use of modern optimization methods with guaranteed convergence to the unique optimal solution. For several special system configurations and/or correlation models, computationally simple closed-form precoders have been also developed. The minimum SER upper-bound precoders perform nearly as good as the minimum 
exact SER precoders, but have lower complexity. The numerical simulations illustrate the excellent performance of the proposed precoders.

\section{APPENDIX A}

\section{BARRIER FUNCTION BASED METHOD}

Given: strictly feasible $\mathrm{x} \in \mathcal{R}_{+}^{N_{\mathrm{t}}}, l:=l^{(0)}>0, \nu>1$ (update parameter), $\epsilon>0$ (tolerance value).

\section{Repeat:}

1) Centering step: Compute $\mathrm{x}^{*}(l)$ by minimizing $l f(\mathbf{x})+\phi(\mathbf{x})$, using the gradient descent method, starting at $x$.

Here $f(\mathbf{x})$ denotes the objective function of (9), $\phi(\mathbf{x}) \triangleq$ $-\sum_{j=1}^{N_{\mathrm{t}}} \log \left(x_{j}\left(1-x_{j}\right)\right)-\log \left(1-\sum_{j=1}^{N_{\mathrm{t}}} x_{j}\right)$, and the gradient $\partial f(\mathbf{x}) / \partial x_{k}$ can be derived as

$$
\begin{aligned}
\frac{\partial f(\mathbf{x})}{\partial x_{k}}= & \int_{0}^{\frac{\pi}{2}} \prod_{\substack{j=1 \\
j \neq k}}^{N_{\mathrm{t}}} \prod_{i=1}^{N_{\mathrm{r}}}\left(1+\varrho_{i j} x_{j}\right)^{-1} \frac{\partial \prod_{i=1}^{N_{\mathrm{r}}}\left(1+\varrho_{i k} x_{k}\right)^{-1}}{\partial x_{k}} d \phi \\
= & -\int_{0}^{\frac{\pi}{2}} \prod_{\substack{j=1 \\
j \neq k}}^{N_{\mathrm{t}}} \prod_{i=1}^{N_{\mathrm{r}}}\left(1+\varrho_{i j} x_{j}\right)^{-1} \prod_{i=1}^{N_{\mathrm{r}}}\left(1+\varrho_{i k} x_{k}\right)^{-2} \\
& \times \sum_{l=1}^{N_{\mathrm{r}}} l\left(\sum_{b_{1}+\ldots+b_{N_{\mathrm{r}}}=l} \prod_{i=1}^{N_{\mathrm{r}}} \varrho_{i k}^{b_{i}}\right) x_{k}^{l-1} d \phi
\end{aligned}
$$

where $\varrho_{i j} \triangleq\left(\tilde{g} / \sin ^{2} \phi\right) \chi_{i} \lambda_{j}$ and $b_{1}, \ldots, b_{N_{\mathrm{r}}}$ is a binary sequence with $b_{i} \in\{0,1\}, i=1, \ldots, N_{\mathrm{r}}$. The expansion $\prod_{i=1}^{L}\left(1+a_{i} x\right)=1+\sum_{l=1}^{L}\left(\sum_{b_{1}+\ldots+b_{L}=l} \prod_{i=1}^{L} a_{i}^{b_{i}}\right) x^{l}$ has been used to derive (22).

2) Update: $\mathrm{x}:=\mathrm{x}^{*}(l)$

3) Stopping criterion: Stop if $\left(N_{\mathrm{t}}+1 / l\right) \leq \epsilon$.

4) Increase: $l:=\nu l$, and go to Step 1 until satisfying the stopping criterion.

\section{APPENDIX B}

\section{COMPLEXITY ANALYSIS}

The complexity of the gradient descend method depends on the number of centering steps and the number of inner iterations for each centering step. The number of centering steps can be computed as $P=\log \left(\left(N_{\mathrm{t}}+1\right) /\left(\epsilon l^{(0)}\right)\right) / \log \nu$. The maximum number of inner iterations can also be computed [15]. Therefore, the log-barrier function based method has bounded (polynomial) overall complexity and is globally convergent.

Comparing the complexity of our precoder (9) to that of the precoder in [11], we find that the latter is based on the fixed-point iterative method of finding $N_{\mathrm{t}} \times N_{\mathrm{t}}$ matrix variables and involves computation of determinant and inversion of $N_{\mathrm{t}} N_{\mathrm{r}} \times N_{\mathrm{t}} N_{\mathrm{r}}$ matrix, as well as computation of $N_{t}^{2}$ integrals. Thus, the complexity per iteration for the precoder in [11] is significantly high than that of our precoder because it is not optimized for the Kronecker case. However, even if the complexity of the precoder in [11] is optimized for the Kronecker case, the fixed-point iteration does not guarantee the convergence to the globally optimal solution since several fixed points may exist.

\section{APPENDIX C}

FAST ALGORITHM FOR THE UPPER-BOUND PROBLEM Initialization: Set $k:=0$ (iteration index), $\mu(0)>0$, and $\beta>0$ (step size).

\section{Repeat:}

1) Find $x_{j}, \forall j$ by solving the following optimization problem

$$
x_{j}^{*}(\mu(k))=\arg \min _{x_{j} \geq 0} \tilde{f}\left(x_{j}\right) \quad j=1, \ldots, N_{\mathrm{t}} .
$$

The solution of (23) is unique due to the strict convexity of $\tilde{f}\left(x_{j}\right)$. This is equivalent to solving the KKT equations (12)-(14). Since the left-hand side of (12) is a decreasing function of $x_{j}$, the IBP given in the end of this Appendix can be applied to find optimal value of $x_{j}$
2) Solve the dual problem to (23), that is

$$
\mu^{*}=\arg \max _{\mu \geq 0} \sum_{j=1}^{N_{\mathrm{t}}} \tilde{f}\left(x_{j}^{*}(\mu)\right)-\mu .
$$

Since the solution to the problem (23) is unique, the dual function in (24) is differentiable and the following gradient method can be used to find optimal value of $\mu: \mu(k+1)=[\mu(k)-\beta(1+$ $\left.\left.\sum_{j=1}^{N_{\mathrm{t}}} \partial \tilde{f}\left(x_{j}^{*}(\mu(k))\right)\right)\right]^{+}$where $\partial \tilde{f}\left(x_{j}^{*}(\mu(k))\right.$ is the gradient of $\tilde{f}\left(x_{j}\right)$ evaluated at $x_{j}^{*}(\mu(k))$.

3) Repeat until convergence. Because of monotonicity, the Lagrange multiplier $\mu(k)$ will converge to the optimal value $\mu^{*}$ as $k \rightarrow \infty$, and $x_{j}(\mu(k)), \forall j$ will also converge to the optimal solutions of (10).

IBP: Suppose we have a decreasing nonlinear monotonic function $h(x)$ and we seek the solution for the nonlinear equation $h(x)=$ $\gamma, x \in\left[x_{l}, x_{u}\right]$. Exploiting the monotonicity of $h(x)$, the solution to the aforementioned nonlinear equation can be found using the following IBP. ${ }^{10}$

1) Set $x=\left(x_{l}+x_{u}\right) / 2$ and reset $x_{l}=x$ if $h(x)>\gamma$, otherwise $x_{u}=x$.

2) Repeat until $h(x)=\gamma$

\section{REFERENCES}

[1] V. Tarokh, H. Jafarkhani, and A. R. Calderbank, "Space-time block codes from orthogonal designs," IEEE Trans. Inf. Theory, vol. 45, no. 5, pp. 1456-1467, Jul. 1999

[2] A. Paulraj, D. A. Gore, R. U. Nabar, and H. Bolcskei, "An overview of MIMO communications-A key to gigabit wireless," Proc. IEEE, vol. 92, no. 2, pp. 198-218, Feb. 2004.

[3] A. Scaglione, P. Stoica, S. Barbarossa, G. B. Giannakis, and H. Sampath, "Optimal designs for space-time linear precoders and decoders," IEEE Trans. Signal Process., vol. 50, no. 5, pp. 1051-1064, May 2002

[4] H. Sampath, P. Stoica, and A. Paulraj, "Generalized linear precoder and decoder design for MIMO channels using the weighted MMSE criterion," IEEE Trans. Commun., vol. 49, no. 12, pp. 2198-2206, Dec. 2001.

[5] G. Jongren, M. Skoglund, and B. Ottersten, "Combining beamforming and orthogonal space-time block coding," IEEE Trans. Inf. Theory, vol. 48, no. 3, pp. 611-627, Mar. 2002.

[6] H. Sampath and A. Paulraj, "Linear precoding for space-time coded systems with known fading correlations," IEEE Commun. Lett., vol. 6 , pp. 239-241, Jun. 2002.

[7] H. R. Bahrami and T. Le-Ngoc, "Precoder design based on correlation matrices for MIMO systems," IEEE Trans. Wireless Commun., vol. 5 , no. 12 , pp. $3579-3587$, Dec. 2006.

[8] S. Zhou and G. B. Giannakis, "Optimal transmitter eigen-beamforming and space-time block coding based on channel correlations," IEEE Trans. Inf. Theory, vol. 49, no. 7, pp. 1673-1690, Jul. 2003.

[9] M. Vu and A. Paulraj, "Optimal linear precoders for MIMO wireless correlated channels with nonzero mean in space-time coded systems," IEEE Trans. Signal Process., vol. 54, no. 6, pp. 2318-2332, Jun. 2006.

[10] H. Shin and J. H. Lee, "Capacity of multiple-antenna fading channels: Spatial fading correlation, double scattering, and keyhole," IEEE Trans. Inf. Theory, vol. 49, no. 10, pp. 2636-2647, Oct. 2003.

[11] A. Hjorungnes and D. Gesbert, "Precoding of orthogonal space-time block codes in arbitrarily correlated MIMO channels: Iterative and closed-form solutions," IEEE Trans. Wireless Commun., vol. 6, no. 3 , pp. 1072-1082, Mar. 2007

[12] L. Liu and H. Jafarkhani, "Applivation of quasi-orthogonal space-time block codes on beamforming," IEEE Trans. Signal Process., vol. 53 , no. 1, pp. 54-63, Jan. 2005.

[13] A. Sezgin, E. A. Jorswieck, and E. Costa, "LDC in MIMO Ricean channels: Optimal transmit strategy with MMSE derection," IEEE Trans. Signal Process., vol. 56, no. 1, pp. 313-328, Jan. 2008.

[14] K. T. Phan, S. A. Vorobyov, and C. Tellambura, "Linear precoders for OSTBC MIMO systems with correlated Rayleigh fading channels based on convex optimization," in Proc. 2nd Int. Workshop Computational Advances in Multi-Sensor Adaptive Processing (CAMSAP), Virgin Islands, Dec. 2007, pp. 221-224.

[15] S. Boyd and L. Vandenberghe, Convex Optimization. Cambridge, U.K.: Cambridge Univ. Press, 2004.

[16] D.-S. Shiu, G. J. Foschini, M. J. Gans, and J. M. Kahn, "Fading correlation and its effect on the capacity of multielement antenna systems," IEEE Trans. Commun., vol. 48, no. 3, pp. 502-513, Mar. 2000.

[17] M. K. Simon and M.-S. Alouini, Digital Communication over Fading Channels. New York: Wiley, 2005.

${ }^{10}$ The complexity of the IBP in one dimension is very low, and the convergence rate is very fast. 